\title{
Avaliação da contribuição do aparelho de amplificação sonora no espectro da neuropatia auditivar um estudo de caso
}

\author{
Assessment of the hearing aids' contribution on the auditory neuropathy \\ spectrum: a case study
}

\author{
Mirela V. Marino'; Erika B. Mantello²; Ana Claudia M. B. dos Reis ${ }^{3}$; Michelle N. Valadão4; Adriana R. T. Anastasio ${ }^{5}$
}

\begin{abstract}
RESUMO
Modelo do estudo: Estudo de caso. Objetivo do estudo: Verificar a contribuição da adaptação do apareIho de amplificação sonora (AAS) em duas pacientes adultas com diagnóstico de Espectro de Neuropatia Auditiva (ENA), em relação às habilidades auditivas e de comunicação. Importância do problema: Indivíduos diagnosticados com ENA apresentam sintomatologia clínica variada com queixa predominante de dificuldade de discriminação de fala, principalmente em ambientes ruidosos e, a literatura, aponta várias possibilidades de tratamento em decorrência dessas diversidades. Metodologia: Foram descritos todos os exames audiológicos, eletroacústicos, eletrofisiológicos e de imagem realizados, bem como avaliação otorrinolaringológica clínica. Participaram deste estudo duas pacientes que passaram por seleção e adaptação de AAS e foram reavaliadas após quatro e 14 meses de uso contínuo dos mesmos, mediante aplicação de teste de percepção de fala (TPF), audiometria em campo livre nas condições com e sem AAS e responderam aos questionários de auto avaliação validados (International Outcame Inventory for Hearing Aids - IOI-HA e Hearing Handicap Inventory for Adults - HHIA). Descrição dos casos: $\mathrm{Na}$ avaliação audiológica, ambas as pacientes apresentaram média dos limiares audiométricos dentro dos padrões de normalidade, porém no caso I observou-se limiares rebaixados a partir da frequência de $3 \mathrm{kHz}$ e no caso II estavam rebaixados os limiares de 250 e $500 \mathrm{~Hz}$; logoaudiometria com resultados incompatíveis com a audiometria; curvas timpanométricas normais e alterações nos reflexos estapedianos bilateralmente; Emissões Otoacústicas presentes bilateralmente; Potencial Evocado Auditivo de Tronco Encefálico ausente bilateralmente com presença de microfonismo coclear; e exames de imagem de orelhas normais. Ambas pacientes receberam adaptação binaural com aparelhos auditivos retroauriculares digitais, devidamente programados de acordo com as características audiológicas e respostas das mesmas frente ao processo de testagem dos dispositivos. Nas avaliações realizadas observou-se para ambas pacientes melhora nos limiares em campo livre com uso de AAS nas frequências rebaixadas na audiometria tonal, TPF com diferença de até $12 \%$ nas condições com e sem AAS e nos questionários atingiram uma média de 24,6 pontos (total de 35 pontos) no IOI-HA e média de 53,6 pontos (total de 100 pontos) no HHIA. A partir desses resultados, foi realizada uma intervenção terapêutica com o objetivo de trabalhar as habilidades auditivas e linguísticas das pacientes, as quais responderam adequadamente à proposta e apresentaram melhora no reconhecimento da fala. Comentários: Ainda não há consenso sobre qual o melhor tratamento para o ENA . A adaptação
\end{abstract}

1. Fonoaudióloga Graduada do Curso de Fonoaudiologia da Faculdade de Medicina de Ribeirão Preto - Universidade de São Paulo (FMRP-USP).

2. Fonoaudióloga Assistente do Curso de Fonoaudiologia FMRP-USP, Doutora em Ciências Médicas pela FMRP-USP.

3,5. Docente do Departamento de Oftalmologia, Otorrinolaringologia e Cirurgia de Cabeça e Pescoço da FMRP-USP

4. Docente do Departamento de Letras da Universidade Federal de Viçosa, Viçosa, Minas Gerais, Brasil.
CORRESPONDÊNCIA: Erika Barioni Mantello Email:erikafga@yahoo.com.br Departamento de Otorrinolaringologia da FMRP-USP. Av. Bandeirantes, 3600, Campus Universitário. 14049-900- Ribeirão Preto/SP Telefone (16) 36022428 Fax (16) 36022860.

Artigo recebido em 08/10/2012 Aprovado para publicação em 28/01/2013 


\begin{abstract}
de AAS é uma das alternativas para os adultos, e no caso em questão, optou-se por iniciar a intervenção com o uso de AAS e acompanhamento fonoaudiológico para ambas as pacientes. Para ambas as pacientes, uma vez adultas e com perda auditiva adquirida, utilizou-se como recurso reabilitativo o AAS e acompanhamento fonoaudiológico, posteriormente, uma vez que não foram obtidos resultados satisfatórios, foram encaminhadas para terapia fonoaudiológica (treinamento auditivo formal). Conclusões: Concluiu-se que, nos casos descritos, o uso do AAS como forma de reabilitação, levou a benefício observado na avaliação audiológica em campo livre, nos testes de percepção de fala e na auto-avaliação do usuário e que, a terapia fonoaudiológica foi fundamental para a melhora da compreensão de fala e a comunicação das pacientes.
\end{abstract}

Palavras-chave: Questionários. Auxiliares de Audição. Transtornos da Percepção Auditiva.

\section{Introdução}

A Neuropatia Auditiva foi descrita, em 1996, como uma anormalidade da função do nervo vestíbulo coclear - VIII par craniano - que gera uma perda da sincronia na condução nervosa do estímulo sonoro e muito provavelmente está relacionada com uma alteração da mielinização dessas fibras. ${ }^{1}$ O local específico dessa disfunção ainda não é conhecido e poderia estar localizado nas Células Ciliadas Internas (CCI), nas sinapses entre as CCI e o VIII par, no VIII par propriamente dito ou em várias dessas estruturas. ${ }^{1,2}$ Essa desordem pode ser encontrada em pacientes de todas as idades. ${ }^{3}$

Em junho de 2008, na Conferência Internacional de Investigação e Conduta de Crianças com Neuropatia Auditiva, alguns pesquisadores entraram em consenso e definiram que a nomenclatura correta para essa desordem seria "Espectro da Neuropatia Auditiva" (ENA), pois reflete melhor o fato de que os portadores dessa desordem podem apresentar uma vasta gama de desordens nas habilidades auditivas. ${ }^{4}$

Os indivíduos com ENA podem apresentar desde limiares tonais dentro da normalidade até uma perda auditiva profunda. ${ }^{1,2,5,6} \mathrm{Em}$ geral, as alterações auditivas são bilaterais, mas há relatos na literatura de casos unilaterais. A discriminação vocal encontra-se, na maioria das vezes, desproporcionalmente mais baixa em relação ao limiar auditivo tonal, sendo incompatível com sua configuração audiológica, além de ser a principal queixa desses pacientes. Podem apresentar também alterações das respostas na pesquisa dos reflexos acústico do músculo estapédio. As Emissões Otoacústicas Transientes (EOA-T) assim como por produto de distorção (EOA-PD) estão presentes na maior parte dos casos, estando o Potencial Evocado Auditivo de Tronco Encefálico (PEATE) ausente ou alterado começando com a onda I e, pode ser eviden- ciado no registro a presença do microfonismo coclear (MC). ${ }^{1,2,5,6,7}$

Para a caracterização do diagnóstico do ENA deve ser realizada uma série de exames audiológicos, eletroacústicos, eletrofisiológicos e de imagem - em indivíduos com alteração específica da audição ou em crianças pertencentes ao grupo de risco para deficiência auditiva, que consiste em complicações na gestação, no parto ou no período neonatal. ${ }^{5,8}$ Embora os fatores de risco para esta desordem não estejam claramente compreendidos, a literatura refere principalmente a prematuridade, a hiperbilirrubinemia, a anóxia e a hemorragia intracraniana. ${ }^{6,8}$

A intervenção frente ao ENA ainda não está bem estabelecida e estendem-se da utilização de aparelhos de amplificação sonora (AAS), o implante coclear (IC) e até o implante de tronco encefálico, ainda pouco conhecido. Nem todos os pacientes diagnosticados com ENA podem ter acesso a esses recursos, sendo em alguns casos até mesmo contra-indicados. Sob outra perspectiva, a terapia fonoaudiológica também figura como um recurso disponível para esses indivíduos somando-se a utilização dos dispositivos anteriormente citados, sendo uma alternativa indispensável e oportuna. Entretanto, a indicação de acompanhamento fonoaudiológico em conjunto com a adaptação do AAS, para esses indivíduos com ENA, deve ser discutida com a família e outros profissionais. ${ }^{8,9}$

Alguns autores acreditam que o uso de AAS pode ser contra-indicado, considerando que a função das Células Ciliadas Externas (CCE) pode estar intacta dependendo do tipo e grau da perda auditiva e, nesses casos, a exposição a um ruído induzido pelo AAS poderia vir a danificá-las. ${ }^{2}$ Por outro lado, os mesmos autores descrevem que alguns indivíduos portadores do ENA são beneficiados com o uso de AAS, no que refere à detecção dos sons, o que não é percebido com relação à discriminação dos mesmos. ${ }^{2}$ 
Visto que não há evidências científicas quanto ao tratamento para tal desordem, que tem como queixa principal a dificuldade de discriminação de fala, principalmente em ambiente ruidoso, justifica-se a realização deste estudo de caso, que teve como objetivo verificar a contribuição da adaptação de AAS associada a intervenção fonoaudiológica em duas pacientes adultas com diagnóstico de ENA, em relação às habilidades auditivas e de comunicação.

\section{Material e Métodos}

Estudo descritivo de duas pacientes diagnosticadas com ENA, atendidas no Serviço de Fonoaudiologia do Departamento de Otorrinolaringologia do Hospital das Clínicas da Faculdade de Medicina de Ribeirão Preto - Universidade de São Paulo (HCFMRP-USP). Esse trabalho foi submetido à análise e aprovado pelo Comitê de Ética do HCFMRPUSP de acordo com o processo de $n^{\circ} 13826 / 2010$.

Para caracterização dos casos foram descritos dados de identificação, queixa e anamnese fonoaudiológica. Posteriormente, foram apresentados todos os exames audiológicos, eletroacústicos e eletrofisiológicos realizados: audiometria tonal liminar, logoaudiometria, imitanciometria, PEATE, EOA-T e/ou EOAPD. Além dos exames de imagem, assim como avaliação clínica otorrinolaringológica.

Após consolidado o diagnóstico do ENA e discussão com equipe multidisciplinar, ambas pacientes foram encaminhadas para seleção, testes e adaptação de AAS. As pacientes puderam experimentar três marcas diferentes de AAS e foram submetidas aos protocolos de rotina ambulatorial, que incluem teste de percepção de fala - IRF e audiometria em campo livre com e sem o AAS, sendo escolhido aquele que ofereceu maior conforto auditivo às usuárias, melhora na percepção de fala e melhores limiares em campo livre com o uso dos mesmos. Realizada também a primeira aplicação do questionário de auto-avaliação Hearing Handicap Inventory for Adult - HHIA, que tem por objetivo quantificar o impacto social e emocional causados pela deficiência auditiva em indivíduos adultos e, também, medir o benefício oferecido pelo AAS na diminuição da restrição de participação (handicap); a análise das respostas do questionário foi realizada considerando-se a pontuação total, classificando em: sem percepção do handicap (entre zero e 16 pontos); percepção leve/moderada do handicap (18 a 42 pontos); severa percepção do handicap (acima de 42 pontos). ${ }^{10}$
Após quatro meses de uso contínuo do dispositivo foi realizada a segunda aplicação do questionário HHIA e a primeira aplicação do questionário International Outcame Inventory for Hearing Aids - IOIHA, que avalia sete domínios considerados importantes para o sucesso do uso do AAS, sob o ponto de vista do indivíduo: uso diário; benefício; limitação de atividade residual; satisfação; restrição de participação residual; impacto nos outros e qualidade de vida. A análise das respostas do questionário foi realizada considerando-se a pontuação total (soma de todas as perguntas) e a pontuação de dois fatores: o fator 1 que diz respeito à interação do paciente com seu AAS, sendo a pontuação variável de 4 a 20 pontos; e o $f a$ tor 2 que diz respeito à interação do paciente com outras pessoas no seu ambiente, sendo a pontuação variável de 3 a 15 pontos. ${ }^{11}$ Assim, uma contagem mais alta é indicativa de uma avaliação positiva do desempenho do AAS. ${ }^{12}$ Além dos questionários também foram realizados o teste de percepção de fala e audiometria em campo livre, para comparar o desempenho das pacientes nas condições com e sem AAS.

Estes procedimentos foram realizados após quatro meses (mais ou menos um mês) de uso dos AAS e foram reaplicados com 14 meses (mais ou menos um mês) após adaptação inicial do AAS, sendo os resultados comparados entre si.

Ao final dos 14 meses de uso do AAS, foi acrescentado ao processo de intervenção, a proposta de realização de atendimentos fonoaudiológicos. Tais atendimentos objetivaram o treinamento das habilidades auditivas.

Os tratamentos dos distúrbios de audição podem ser os mais variados de acordo com o quadro clínico e o impacto da deficiência e/ou perda auditiva sobre sua funcionalidade. Para ambas as pacientes, uma vez adultas e com perda auditiva adquirida, utilizou-se inicialmente como recurso reabilitativo o AAS e acompanhamento fonoaudiológico, quatro sessões, mensais, individuais, acompanhamento este previsto na Portaria de Saúde Auditiva ${ }^{13,14}$; posteriormente, uma vez que não foram obtidos resultados satisfatórios, foram encaminhadas para terapia fonoaudiológica semanal individual (treinamento auditivo formal).

\section{Relato de Caso}

\section{CASO 1}

Sujeito 1, 27 anos, gênero feminino, etnia mulata, profissão atendente de supermercado, solteira, sem alterações físicas anatômicas estruturais percebidas 
por meio de inspeção física. Foi encaminhada para o Serviço de Fonoaudiologia do HCRP por apresentar queixa de dificuldades na fala e na compreensão. Quanto aos dados de anamnese fonoaudiológica, paciente nasceu a termo, parto normal, sem intercorrências; desenvolvimento da linguagem e motor adequados. Aos 7 anos de idade apresentou episódio de crise convulsiva, a qual foi controlada com medicamento (segundo informações colhidas do paciente), porém após esse fato começou com a queixa de dificuldade auditiva, que se mantém desde então. Atualmente, nega problemas de saúde e uso diário de medicamento.

As informações obtidas no prontuário mostram que na avaliação clínica otorrinolaringológica foram observadas otoscopia normal bilateral, nariz, laringe, região facial e cervical sem alterações, septo nasal centrado, seios paranasais e orofaringe sem alterações, exame dos nervos cranianos adequado, testes de Romberg e Untemberg negativos e ausência de nistagmo.

Na avaliação audiológica apresentou: audiometria tonal limiar com média dos limiares audiométricos (de $500 \mathrm{a} 2 \mathrm{kHz}$ ) dentro dos padrões de normalidade, de acordo com a classificação de Davis e Silverman ${ }^{15}$ com queda nas frequências agudas a partir de $3 \mathrm{kHz}$ na orelha esquerda e a partir de $4 \mathrm{kHz}$ na orelha direita (Tabela 1). Logoaudiometria com resultados de IRF incompatíveis com os limiares auditivos; orelha direita com $72 \%$ de acertos para monossílabos e $80 \%$ para dissílabos, orelha esquerda com $76 \%$ de acertos para monossílabas e $88 \%$ para dissílabas, sendo este desempenho abaixo do esperado. Imitânciometria com curva timpanométrica tipo A bilateralmente, indicando mobilidade normal do sistema tímpano-ossicular, de acordo com Jerger ${ }^{16}$ e alteração nas respostas da pesquisa dos reflexos acústicos, com nível de sensação elevado nas frequências de 500 e $1 \mathrm{kHz}$ e ausência para 2 e $4 \mathrm{kHz}$ em ambas orelhas. EOA-T com presença de resposta em todas as bandas de frequência bilateralmente. PEATE, utilizando estímulo click, com ausência das ondas I, III e V a 100 dBNA e presença de microfonismo coclear.

Os laudos dos exames de imagem (tomografia computadorizada de crânio e ressonância magnética dos ouvidos) apresentaram resultados dentro dos padrões de normalidade.

Paciente recebeu então, diagnóstico do ENA e foi encaminhada para teste e adaptação de AAS, no qual se optou por adaptação binaural, classe C SUS (digital). Essa escolha foi baseada nas características físicas e eletroacústicas dos aparelhos, pois oferecem um melhor ajuste por bandas e canais, modelo miniretroauricular, com molde do tipo canal e material rígido (acrílico), ventilado. Em avaliação do IRF à viva voz, para dissílabos, paciente obteve $80 \%$ de acerto sem AAS e sem leitura orofacial (LOF) e $92 \%$ de acerto na situação com AAS e sem LOF. Na primeira aplicação do questionário de auto-avaliação HHIA, obteve a pontuação total de $60 / 100$ pontos, que carac-

\section{Tabela 1}

Limiares audiométricos encontrados na Audiometria Tonal Liminar para o Caso I

\begin{tabular}{|c|c|c|c|c|}
\hline & \multicolumn{2}{|c|}{$O D$} & \multicolumn{2}{|c|}{$O E$} \\
\hline & VA & VO & VA & VO \\
\hline $250 \mathrm{~Hz}$ & $35 \mathrm{~dB}$ & - & $40 \mathrm{~dB}$ & - \\
\hline $500 \mathrm{~Hz}$ & $20 \mathrm{~dB}$ & - & $20 \mathrm{~dB}$ & -_- \\
\hline $1000 \mathrm{~Hz}$ & $20 \mathrm{~dB}$ & _-____ & $15 \mathrm{~dB}$ & . \\
\hline $2000 \mathrm{~Hz}$ & $25 \mathrm{~dB}$ & $25 \mathrm{~dB}$ & $20 \mathrm{~dB}$ & - \\
\hline $3000 \mathrm{~Hz}$ & $25 \mathrm{~dB}$ & $25 \mathrm{~dB}$ & $50 \mathrm{~dB}$ & $45 \mathrm{~dB}$ \\
\hline $4000 \mathrm{~Hz}$ & $55 \mathrm{~dB}$ & $55 \mathrm{~dB}$ & Ausente & Ausente \\
\hline $6000 \mathrm{~Hz}$ & $90 \mathrm{~dB}$ & -_____ & Ausente & - \\
\hline $8000 \mathrm{~Hz}$ & $90 \mathrm{~dB}$ & - & Ausente & \\
\hline
\end{tabular}

OD: orelha direita; OE: orelha esquerda; VA: via aérea; VO: via óssea; dB: decibéis nível de audição.

**Utilizado mascaramento (NarrowBand Noise- NB) na OD, nas frequências de 2,3 e 4KHz, em VO de 60 a 80 dBNA. 
teriza uma severa percepção do handicap em relação à perda auditiva.

No primeiro retorno, após 4 meses de uso dos aparelhos, foi realizada a primeira aplicação do questionário de auto-avaliação IOI-HA. No que se refere à pontuação total, obteve $27 / 35$ pontos, quanto ao $f a$ tor 1 atinge 18/20 pontos e quanto ao fator 2 atingiu $9 / 15$ pontos. Tais resultados mostraram uma avaliação positiva quanto ao nível de satisfação do usuário de AAS, tanto na percepção do paciente quanto na relação com as outras pessoas. Na segunda aplicação do questionário de auto-avaliação HHIA, no qual foi considerada a pontuação total com 56/100 pontos, mantém uma severa percepção do handicap. Em teste de percepção de fala, para dissílabos, observou-se manutenção dos mesmos resultados obtidos no dia do teste inicial. Na audiometria em campo livre com os AAS(s) testados individualmente, em volume habitual de uso, houve uma variação média de $20 \mathrm{~dB}$ na diferença entre as situações com e sem AAS para as frequências audiométricas nas quais ocorrem perda auditiva (3 e $4 \mathrm{KHz}$ ).

No segundo retorno, após 14 meses de uso dos aparelhos, foi realizada a segunda aplicação do questionário de auto-avaliação IOI-HA, considerando a pontuação total, na qual obteve $27 / 35$ pontos e também, quanto ao fator 1 atingindo 14/20 pontos e o fator 2 com 13/15 pontos. Tais resultados mostraram que a pontuação total se manteve igual em relação à primeira aplicação do questionário. $\mathrm{O}$ teste de percepção de fala, realizado com monossílabos e dissílabos, não apresentou diferença nas situações com e sem AAS. Realizada ainda, audiometria em campo livre para a pesquisa dos limiares auditivos com e sem os AAS(s), os quais foram testados individualmente e em volume habitual de uso. Observou-se as mesmas variações encontradas no primeiro retorno.

\section{CASO 2}

Sujeito 2, 45 anos, gênero feminino, etnia branca, casada, não trabalha, sem alterações físicas anatômicas estruturais percebidas por meio de inspeção física. Encaminhada para o Serviço de Fonoaudiologia do HCRP por apresentar queixa de hipoacusia e dificuldades de compreensão de fala. Quanto aos dados de anamnese, paciente nasceu a termo, parto normal, sem intercorrências; a mesma não soube relatar se houve algum tipo de intercorrência no decorrer do seu desenvolvimento, tanto motor quanto de linguagem. Há aproximadamente 25 anos apresentou episódio de prurido e pressão em ambas as orelhas com duração aproximada de uma semana, quando iniciou com queixa de hipoacusia e zumbido bilateral. Atualmente, paciente nega problemas de saúde e uso diário de medicamento.

Os dados do prontuário referentes à avaliação clínica otorrinolaringológica demonstraram otoscopia normal bilateralmente, nariz, laringe, região facial e cervical sem alterações, septo nasal centrado, seios paranasais e orofaringe sem alterações, exame dos nervos cranianos adequado, testes de Romberg e Untemberg negativos e ausência de nistagmo.

$\mathrm{Na}$ avaliação audiológica apresentou: audiometria tonal limiar com média dos limiares audiométricos (de 500 a $2 \mathrm{kHz}$ ) dentro dos padrões de normalidade de acordo com a classificação de Davis e Silverman, ${ }^{15}$ com configuração ascendente bilateralmente (Tabela 2). Logoaudiometria com resultados de IRF incompatíveis com os limiares auditivos; orelha direita com $76 \%$ de acertos para monossílabos e $80 \%$ para dissílabos, orelha esquerda com $80 \%$ de acertos para monossílabas e $84 \%$ para dissílabas, sendo este desempenho abaixo do esperado. Imitanciometria com curva timpanométrica tipo Ad bilateralmente, indicando sistema tímpano-ossicular hiper-móvel, de acordo com Jerger ${ }^{16}$ e ausência de respostas, até a saída máxima do equipamento, na pesquisa dos reflexos acústicos contralaterais bilateralmente. EOA-T e EOA-PD com presença de resposta em todas as bandas de frequência bilateralmente. PEATE, utilizando estímulo click, com ausência das ondas I, III e V a 90 dBNA e presença de microfonismo coclear.

Os laudos dos exames de imagem (tomografia computadorizada de crânio e ressonância magnética dos ouvidos) apresentaram resultados dentro dos padrões de normalidade.

Paciente diagnosticada com ENA e encaminhada para teste e adaptação de AAS. Assim, como relatado para a paciente anterior, optou-se pela escolha de adaptação binaural de AAS(s) classe C SUS (digital) por oferecer um melhor ajuste por bandas e canais, modelo retroauricular e molde do tipo canal, material rígido (acrílico). Realizado IRF a viva voz, para dissílabos, no qual paciente obteve $80 \%$ de acerto sem AAS e sem LOF e $88 \%$ de acerto na situação com AAS e sem LOF e a primeira aplicação do questionário de auto-avaliação HHIA, no qual foi considerada a pontuação total com $56 / 100$ pontos que caracteriza uma severa percepção do handicap em relação à sua perda auditiva.

No primeiro retorno, após quatro meses de uso do AAS, foi realizada a primeira aplicação do questio- 


\section{Tabela 2}

Limiares audiométricos encontrados na Audiometria Tonal Limiar para o Caso II

\begin{tabular}{llcccc}
\hline & \multicolumn{2}{c}{ OD } & & \multicolumn{2}{c}{ OE } \\
\cline { 2 - 5 } \cline { 5 - 5 } & VA & VO & VA & VO \\
\hline $250 \mathrm{~Hz}$ & $45 \mathrm{~dB}$ & ---- & $60 \mathrm{~dB}$ & --- \\
$500 \mathrm{~Hz}$ & $40 \mathrm{~dB}$ & $40 \mathrm{~dB}$ & $45 \mathrm{~dB}$ & $40 \mathrm{~dB}$ \\
$1000 \mathrm{~Hz}$ & $20 \mathrm{~dB}$ & $20 \mathrm{~dB}$ & $20 \mathrm{~dB}$ & $20 \mathrm{~dB}$ \\
$2000 \mathrm{~Hz}$ & $10 \mathrm{~dB}$ & $10 \mathrm{~dB}$ & $15 \mathrm{~dB}$ & $10 \mathrm{~dB}$ \\
$3000 \mathrm{~Hz}$ & $10 \mathrm{~dB}$ & $10 \mathrm{~dB}$ & $10 \mathrm{~dB}$ & $10 \mathrm{~dB}$ \\
$4000 \mathrm{~Hz}$ & $15 \mathrm{~dB}$ & $15 \mathrm{~dB}$ & $20 \mathrm{~dB}$ & $15 \mathrm{~dB}$ \\
$6000 \mathrm{~Hz}$ & $15 \mathrm{~dB}$ & - & $20 \mathrm{~dB}$ & --- \\
$8000 \mathrm{~Hz}$ & $30 \mathrm{~dB}$ & - & $20 \mathrm{~dB}$ & -- \\
\hline
\end{tabular}

OD: orelha direita; OE: orelha esquerda; VA: via aérea; VO: via óssea; dB: decibéis nível de audição; Hz: hertz.

nário de auto-avaliação IOI-HA, na qual obteve 22/35 pontos no total, fator 1 atingiu 13/20 pontos e ofator 2 obteve 9/15 pontos. Tais resultados mostraram uma avaliação razoavelmente positiva quanto ao desempenho dos AAS(s), tanto na percepção da paciente, quanto na relação com as outras pessoas. Realizada a segunda aplicação do questionário de auto-avaliação HHIA, no qual obteve a pontuação total de 50/100 pontos, o que aponta para a manutenção de uma severa percepção do handicap. Em teste de percepção de fala, realizado com dissílabos, a viva voz e sem LOF paciente apresentou $92 \%$ de acertos nas condições com e sem os AAS(s). Realizada também, audiometria em campo livre para a pesquisa dos limiares com e sem os AAS(s), os quais foram testados em volume habitual de uso, observou-se que houve uma variação média de $5 \mathrm{~dB}$ na diferença entre as situações com e sem AAS na frequência de $500 \mathrm{~Hz}$.

No segundo retorno, após 14 meses de uso dos AAS, foi realizada a segunda aplicação do questionário de auto-avaliação IOI-HA, na qual obteve 19/35 pontos no total, fator 1 atingindo $13 / 20$ pontos e o fator 2 com $6 / 15$ pontos. Tais resultados mostraram uma avaliação pior em relação à anterior, quanto ao desempenho com o uso das próteses auditivas, principalmente no que se refere à relação da paciente com as outras pessoas no seu ambiente. No teste de percepção de fala, realizado à viva voz e sem LOF, na tarefa com monossílabos obteve $48 \%$ de acertos sem o AAS e $60 \%$ com os mesmos; e na tarefa com dissí- labos obteve $76 \%$ de acertos sem o AAS e $80 \%$ com os mesmos. Realizada ainda, audiometria em campo livre para a pesquisa dos limiares com e sem os AAS(s), os quais foram testados individualmente e em volume habitual de uso, na qual se observou uma diferença de 15 dBNA na frequência de $500 \mathrm{~Hz}$ para ambas orelhas.

A partir dos achados das avaliações, as pacientes foram encaminhadas para terapia fonoaudiológica, que consistiram em dez atendimentos semanais, com duração de 40 minutos cada, cujo objetivo foi o treinamento das habilidades auditivas.

Foram enfatizadas as habilidades auditivas para tarefas cada vez mais complexas por meio de atividades intensivas envolvendo escuta monótica e dicótica para o trabalho de discriminação, reconhecimento, compreensão e figura-fundo auditivo.

O trabalho foi direcionado considerando o critério de complexidade do treino, ou seja, das atividades mais simples às mais complexas, portanto, durante os atendimentos procurou-se reduzir as pistas e aumentar a dificuldade de escuta, por meio de atividades desafiadoras do tipo: redução da intensidade do sinal, apresentação de sons competitivos, distorção do sinal verbal e aumento da velocidade da fala.

A princípio ambas as pacientes apresentaram dificuldades nas atividades realizadas, porém conforme intensificado o treinamento, conseguiram alcançar os objetivos propostos.

O trabalho de reconhecimento auditivo para 
sons verbais esteve associado à apresentação de uma sequência de três palavras (processamento temporal), iniciando com palavras em conjunto fechado. Como tarefa, a paciente deveria apontar a palavra escutada. No início do treino as pacientes não conseguiam realizar o reconhecimento das palavras auditivamente, necessitavam da LOF. Em seguida, o treinamento auditivo passou a exigir atividades gradativamente mais complexas, envolvendo reconhecimento auditivo em conjunto aberto, compreensão auditiva de sons de fala em ambientes silenciosos e com ruído de fundo. As pacientes apresentaram evolução em todos os aspectos trabalhados, alcançando os objetivos propostos nas dez sessões.

Na última sessão de atendimento fonoaudiológico, as pacientes ainda referiam queixa de dificuldade de compreensão de fala, principalmente em ambientes ruidosos. Ambas receberam orientações quanto à evolução alcançada neste período, sendo apresentados os resultados por elas alcançados se- manalmente. Também foram realizadas orientações aos acompanhantes para intensificar o treinamento em casa em diversas situações relacionadas ao cotidiano das mesmas.

Após essa intervenção fonoaudiológica, as pacientes foram reavaliadas, o que coincidiu com um período de 18 meses de uso de AAS, a fim de se verificar alterações no desempenho dos testes após a terapia. Pode-se observar que os resultados dos exames mantiveram-se semelhantes aos encontrados nas avaliações anteriores.

Os resultados obtidos nas aplicações dos questionários de auto-avaliação de ambas as pacientes foram sintetizados nas Tabelas 3 e 4, para uma melhor visualização da evolução ocorrida ao longo do tempo com o uso dos AAS(s). Considerando que para o questionário IOI-HA uma pontuação mais alta é indicativa de um resultado positivo (maior satisfação), enquanto que para o HHIA a pontuação mais baixa é indicativa de um resultado positivo (menor handicap).

\section{Tabela 3}

Síntese dos resultados obtidos nas aplicações dos questionários de auto-avaliação do Caso I.

\begin{tabular}{|c|c|c|c|c|c|c|}
\hline & \multicolumn{3}{|c|}{ IOI-HA } & \multicolumn{3}{|c|}{ HHIA } \\
\hline & $\mathbf{T}$ & F1 & F2 & $\mathbf{T}$ & $\mathbf{E}$ & $\mathbf{S}$ \\
\hline Antes do AAS & & & & 60 & 34 & 26 \\
\hline Retorno 4 meses & 27 & 18 & 9 & 56 & 18 & 38 \\
\hline Retorno 14 meses & 27 & 14 & 13 & & & \\
\hline Retorno 18 meses & 28 & 15 & 13 & 52 & 18 & 34 \\
\hline
\end{tabular}

T: pontuação total; F1: sub-escala fator 1; F2: sub-escala fator 2; E: sub-escala emocional; S: sub-escala social.IOI-HA: International Outcame Inventory for Hearing Aids; HHIA: Hearing Handicap Inventory Adults.

\section{Tabela 4}

Síntese dos resultados obtidos nas aplicações dos questionários de auto-avaliação do Caso II.

\begin{tabular}{|c|c|c|c|c|c|c|}
\hline & \multicolumn{3}{|c|}{ IOI-HA } & \multicolumn{3}{|c|}{ HHIA } \\
\hline & $\mathbf{T}$ & F1 & F2 & $\mathbf{T}$ & $\mathbf{E}$ & $\mathbf{S}$ \\
\hline Antes do AAS & & & & 56 & 28 & 28 \\
\hline Retorno 4 meses & 22 & 13 & 9 & 50 & 28 & 22 \\
\hline Retorno 14 meses & 19 & 13 & 6 & - & & \\
\hline Retorno 18 meses & 25 & 12 & 13 & 46 & 24 & 22 \\
\hline
\end{tabular}

T: pontuação total; F1: sub-escala fator 1; F2: sub-escala fator 2; E: sub-escala emocional; S: sub-escala social.IOI-HA: International Outcome Inventory for Hearing Aids; HHIA: Hearing Handicap Inventory Adults. 


\section{Discussão}

De acordo com Silva e Araújo ${ }^{17}$ o ENA ainda não possui etiologias definidas. Alguns autores apontam que os possíveis fatores etiológicos para essa desordem seriam a prematuridade,,${ }^{5,18}$ herança genética (recessiva/dominante), uma neuropatia sensorial motora hereditária, ${ }^{19,20}$ neuropatias perifericas, anóxia e hiperbilirrubinemia. ${ }^{3,7,21,22}$ Não foram identificados, na história clínica das pacientes da pesquisa fatores que poderiam estar relacionados às etiologias descritas nessas literaturas. Assim, faz-se necessários investimentos que identifiquem os fatores etiológicos envolvidos nessa condição fisiopatológica ainda não completamente definida.

Ambas as pacientes do estudo não relataram a presença de nenhum dos fatores supracitados em sua história desenvolvimental. O único achado, descrito no prontuário do caso 1 , foi um episódio de convulsão aos sete anos de idade, o qual foi relacionado pela paciente com o início da queixa auditiva.

Os achados dessa pesquisa em relação aos exames audiológicos das pacientes descritas vão de acordo com a literatura pesquisada, pois segundo alguns autores a característica básica dos portadores do ENA é a incompatibilidade dos resultados audiológicos, com um baixo IRF para a configuração audiológica encontrada na audiometria tonal, presença de respostas nas EOAs e ausência ou anormalidade nas respostas do PEATE ${ }^{1,2,3}$. A literatura ainda traz que a redundância, considerada um fator facilitador na perda coclear, para o ENA gera uma piora no reconhecimento da fala ${ }^{5}$, porém em nosso estudo observou-se que a redundância facilitou o reconhecimento da fala nos testes da logoaudiometria e também no treino em terapia, o que provavelmente decorreu das estratégias utilizadas durante as sessões de terapia.

Para ambas as pacientes deste estudo utilizouse como recurso reabilitativo o AAS e a terapia fonoaudiológica, que segundo Rondina e Matas ${ }^{8}$ são possíveis intervenções fonoaudiológicas frente a esses casos. Ainda não há consenso sobre qual o melhor tratamento para o ENA. O uso do AAS tem sido uma das alternativas para os adultos.

Nas rotinas da fonoaudiologia clínica, os indivíduos portadores de deficiências auditivas periféricas, que não o ENA, ao começarem a fazer uso de AAS devem passar por uma etapa de verificação e validação do processo de adaptação das próteses, que é realizada por meio de procedimentos como a verifica- ção do ganho, testes de reconhecimento de fala e questionários de auto-avaliação. Tais procedimentos têm o objetivo de verificar se as características projetadas na etapa de seleção foram realmente obtidas. ${ }^{23}$

Optou-se por utilizar neste trabalho os mesmos procedimentos, já descritos na literatura, para verificação da adaptação do AAS. Entretanto, não foram encontrados trabalhos que utilizassem de questionários de auto-avaliação no processo de verificação e validação do AAS em portadores do ENA.

Os achados obtidos nos retornos de ambas as pacientes (aos quatro e aos 14 meses de uso dos AAS) mostram que o uso dos dispositivos contribuíram para melhor detecção do som. Estes achados puderam ser comprovados diante da melhora dos limiares audiométricos com AAS em campo livre nas frequências anteriormente alteradas. Além disso, houve melhora na percepção de fala. Quanto aos questionários de auto-avaliação, as pacientes, embora não estivessem plenamente satisfeitas, desejavam manter o uso dos AAS por apresentarem benefício com o uso dos mesmos (constatado no IOI-HA) e apresentarem uma diminuição do handicap (constatado no HHIA).

$\mathrm{Hood}^{3}$ relatou que os pacientes adultos portadores do ENA geralmente referem pouco ou até nenhum benefício com o AAS. A autora indicou a utilização de aparelhos de alta qualidade e baixo ganho para minimizar possíveis efeitos deletérios da amplificação, além de um monitoramento. Nesta pesquisa, foram utilizados AAS(s) com tais características, conforme sugerido por essa autora. Além disso, as pacientes passaram por monitoramento nos quais relataram benefício com o uso da amplificação. Outro fator de melhora, também relatado na literatura, é a aclimatização, período em que ocorre uma melhora progressiva das habilidades auditivas de detecção e reconhecimento de sons de fala decorrente das novas pistas de fala disponíveis ao usuário da amplificação. ${ }^{24} \mathrm{Sa}-$ bendo que essa melhora ocorre ao longo do tempo, mas que só acontece após um período de seis a doze (12) semanas de uso do AAS, ${ }^{24}$ as avaliações foram realizadas após o período indicado, sendo a primeira com quatro meses de uso, um período mínimo para que ocorra tal fenômeno, e a segunda com quatorze (14) meses de uso. Além dos achados encontrados nos exames, observou-se a diminuição do handicap, no HHIA, no que se refere às situações sem uso anterior de AAS (no dia da testagem) e após três meses de uso contínuo do dispositivo.

Este achado vai de acordo com relatos de pa- 
cientes que foram igualmente beneficiados com o uso das próteses auditivas, pois Madden ${ }^{6}$ e Dell' Aringa ${ }^{25}$ indicam o uso dos AAS(s) para pacientes com o ENA e confirmam que este dispositivo melhorou em até $30 \%$ o reconhecimento de fala de seus usuários.

Diante da satisfação parcial das pacientes com o recurso de reabilitação escolhido até então, enfatizou-se, a partir daí, um treinamento fonoaudiológico para estimulação das habilidades auditivas prejudicadas. Vários autores são favoráveis à terapia fonoaudiológica nos pacientes com o ENA, sendo esta, em conjunto ou não com o uso de dispositivos (AAS, IC, sistema FM), indicada para o treinamento das habilidades linguísticas e auditivas ${ }^{2,3,7,8,9}$. Observou-se melhora de ambas as pacientes no desempenho das atividades propostas durante a terapia, uma vez que estas foram se tornando gradativamente mais complexas. Porém a condição ideal para a comunicação das pacientes ainda não foi atingida, pois alguma queixa ainda se mantém. Ambas receberam orientações de estratégias que podem ajudar no treinamento dessas habilidades possibilitando a melhora na compreensão da fala em situações de vida diária. Dentre elas temos uso da LOF, que segundo Oliveira ${ }^{9}$ é uma das estratégias de comunicação mais utilizadas por indivíduos com o ENA. Esses mesmos autores relataram que ao utilizarem o AAS como alternativa para compensar o déficit auditivo, os indivíduos com ENA diminuíram o emprego de estratégias facilitadoras da comunicação9. Este recurso foi utilizado também na terapia das pacientes estudadas.

Ao comparar os resultados das três aplicações dos questionários de auto-avaliação observam-se mudanças positivas em relação ao benefício trazido pelo AAS e diminuição do handicap após o uso deste dispositivo. Estes resultados apontam que o recurso do AAS foi considerado positivo pelas pacientes e após intervenção terapêutica, a auto-percepção do benefício e diminuição do handicap foi ainda maior.
Os achados deste estudo sugerem que, nesses casos, se ao utilizar o AAS for verificado algum benefício, quer seja nos testes de avaliação em campo livre, nos testes de percepção de fala ou na auto-avaliação do usuário, este deve ser considerado positivo e a partir daí novas estratégias devem ser somadas ou modificadas, a fim de melhorar a compreensão de fala e a comunicação deste indivíduo.

\section{Considerações Finais}

Nos casos descritos, o uso do AAS, como forma de reabilitação gerou benefício para as pacientes. Foram observadas melhora na detecção dos sons na avaliação audiológica em campo livre, melhora percentual nos testes de percepção de fala e também na auto-percepção das usuárias nos questionários aplicados, nas três avaliações realizadas, auxiliando os sujeitos na auto-percepção de seus reais handicap e benefícios do processo de intervenção (AAS e terapia fonoaudiológica) bem como para subsidiar o planejamento do processo terapêutico.

Há de se considerar que a terapia fonoaudiológica também colaborou para melhora das habilidades auditivas e desempenho na comunicação das pacientes.

Deve-se ressaltar que ao planejar a intervenção fonoaudiológica para pacientes com ENA, esta deve ser individualizada, em virtude da heterogeneidade dos casos, assim como foi aqui descrito. Além disso, não é possível generalizar os resultados obtidos, sendo necessário mais pesquisas que investiguem acerca dos benefícios do uso do AAS na percepção de fala em situações de ruído, da indicação do IC para esses casos, bem como do sistema FM e da terapia fonoaudiológica. A utilização destes recursos, aplicados em casos de ENA, deve ser avaliada isoladamente e em conjunto uma vez que o processo de reabilitação nestes casos ainda é um desafio para os profissionais.

\footnotetext{
ABSTRACT

Model study: Case study. Objective: This study verified the contribution of the hearing aids on two adult patients diagnosed with auditory neuropathy spectrum (ANS), related to their communication and listening skills. Importance of the problem: Patients diagnosed with the ANS exhibit varied clinical symptoms with predominant complain of difficulty understanding speech, particularly in the presence of noise, and the literature suggests several possibilities of treatment due to these differences. Methodology: We described all audiological, electroacoustic, electrophysiological and imaging tests performed, as well as otorhinolaryngological evaluation. The study included two patients, who have gone through selection and adaptation with the hearing aids and were evaluated after four and fourteen months of continuous
} 
use of the device with the application of speech perception test, audiometry in free field with and without the device and they answered to the questionnaires of self-evaluation validated (International Outcame Inventory for Hearing Aids - IOI-HA e Hearing Handicap Inventory for Adults - HHIA). Description os cases: On the audiological assessment, both patients had average audiometric thresholds within normal limits, however for case 1 we observed downgraded thresholds from the frequency of $3 \mathrm{kHz}$ and for case 2 were downgraded thresholds of 250 and $500 \mathrm{~Hz}$; logoaudiometry with inconsistent results with audiometry; normal tympanometric curves and impairment in acoustic reflexes bilaterally; presence of otoacustic emissions bilaterally; absence of auditory brainstem evoked potentials and presence of cochlear microphonic bilaterally; and normal image tests. Both patients received binaural adaptation with hearing aids behind the ear digital, properly regulated based on the audiological features and on the answers given on the process of testing the device. On the assessments was noted that both patients had the threshold improved on free field audiometry with the hearing aids on frequencies downgraded on audiometry, speech perception test with $12 \%$ of difference with and without de device and on IOI-HA questionnaire they had an average of 24,6 points (35 total points), on HHIA questionnaire they had an average of 53,6 points (100 total points). With these results, therapeutic intervention was performed with the aim of working the listening and language skills of the patients, who responded rightly to the proposal and showed improvement in speech recognition. Comment: There is still no consensus on the best treatment for ANS. The use of hearing aids have been one of the options for adults, and on this case, we chose to start the intervention with the use of hearing aids and speech therapy for both patients. For the two patients, once adults and with acquired hearing loss, we used as rehabilitative remedy the hearing aids and after the therapeutic intervention, since no satisfactory results were obtained, they were referred for speech terapy (formal auditory training). Conclusions: We conclude that, in the cases described, the use of the hearing aids as a method of rehabilitation, provided benefit observed on free field audiometry, speech perception tests and on self-evaluation of the user, and the therapy was fundamental in improving speech understanding and communication of patients.

Key-words: Questionnaires. Hearing Aids. Auditory Perceptual Disorders.

\section{Referências Bibliográficas}

1. Starr A, Picton TW, Sininger Y, Hood LJ, Berlin Cl. Auditory neuropathy. Brain. 1996; 119: 741-53.

2. Doyle KJ, Sininger Y, Starr A. Auditory neuropathy in childhood. Laryngoscope. 1998; 108: 1374-7.

3. Hood LJ. Auditory neuropathy: what is it and what can we do about it? Hear J. 1998;51: 10-7.

4. Pearce W, Martin RL. On auditory neuropathy, aka auditory neuropathy spectrum. Hear J. 2009; 62: 38-9.

5. Spinelli M, Fávero-Breurl ML, Silva CMS. Neuropatia Auditiva: aspectos clínicos, diagnósticos e terapêuticos. Rev Bras Otorrinolaringol. 2001; 67: 863-7.

6. Madden C, Rutter M, Hilbert L, Greinwald Junior JH, Choo DI. Clinical and Audiological Features in Auditory Neuropathy. Arch Otolaryngol Head Neck Surg. 2002; 128: 1026-30.

7. Hood LJ. Auditory neuropathy/auditory dys-synchrony: New insights. Hear J. 2002; 55: 10,14,17-8.

8. Rondina C, Matas CG. Neuropatia Auditiva: estudo de caso. Acta Otolayngol. 2006; 24: 10-7.

9. Oliveira JRM, Kuchar J, Valarelli TP. Estratégias de Comunicação Utilizada por Indivíduos com Neuropatia/Dessincronia Auditiva. Rev CEFAC. 2011; 13: 85-91.

10. Lima II, Aiello CP, Ferrari DV. Correlações Audiométricas do Questionário de Handicap Auditivo para Adultos. Rev CEFAC. 2011; 13: 496-503.

11. Cox RM, Alexander GC. The international outcome inventory for hearing aids (IOI-HA): psychometric properties of the English version. Int J Audiol. 2002; 41: 30-5.
12. Assayag FHM, Russo ICP. Avaliação subjetiva do benefício e dos efeitos proporcionados pelo uso de amplificação sonora em indivíduos idosos. Distúrb Comun. 2006; 18: 383-90.

13. BRASIL. Secretaria de Assistência a Saúde. Ministério da Saúde. Portaria № 587, 07 de outubro de 2004. Que determina que as Secretarias de Estado da Saúde dos estados adotem as providências necessárias à organização e implantação das Redes Estaduais de Atenção à Saúde Auditiva e de outras providências. Diário Oficial da República Federativa do Brasil. p. 105, 11 de outubro, 2004. Portaria № 589 SAS/MS de 08/10/2004 - pág. 07, 08/10/2004, seção 1, pág 58, 08/12/2004 (D.O.U.).

14. BRASIL. Secretaria de Assistência a Saúde. Ministério da Saúde. Portaria № 589, 08 de outubro de 2004. Que versa sobre a necessidade de qualificação dos processos de avaliação diagnóstica, tratamento clínico, seleção, adaptação e fornecimento de aparelhos de amplificação sonora individual, assim como acompanhamentos e terapia fonoaudiológica. Diário Oficial da República Federativa do Brasil. p. 58, 08 de dezembro, 2004.

15. Davis $\mathrm{H}$, Silverman RS. Hearing and deafness. $3^{\mathrm{a}}$ ed. Nova York: Rinehart \&Wiston; 1970.

16. Jerger J. Clinical experience with impedance audiometry. Arch Otolaryngol. 1970; 92: 311-24.

17. Silva RCL, Araújo SG. Os resultados do implante coclear em crianças portadoras de Neuropatia Auditiva: revisão de literatura. Rev Soc Bras Fonoaudiol. 2007; 12: 252-7.

18. Berlin $\mathrm{Cl}$. Auditory neuropathy. Using OEAs and ABR from screening to management. Semin Hear. 1999; 20: 307-15. 
19. De Melo TM, Moreti ALM, Bevilacqua MC. Avaliação da produção de fala em crianças deficientes auditivas usuárias de Implante Coclear Multicanal. Rev Soc Bras Fonoaudiol. 2008; 13: $45-51$.

20. Sharma A, Cardon G, Henion K, Roland P. Cortical maturation and behavioral outcomes in children with auditory neuropathy spectrum disorder. Int J Audiol. 2011; 50: 98-106.

21. Martinho AC, Lewis DR. Achados audiológicos em crianças com hiperbilirrubinemia neonatal: um enfoque na neuropatia auditiva/dessincronia auditiva. Distúrb Comun. 2005; 17: 18390.

22. Ogeda ECM, Matas CG. Neuropatia auditiva por Kernicterus: estudo de caso. Pró-Fono. 2002; 14: 247-52.
23. Matas CG, lório MCM. Verificação e Vlidação do Processo de Seleção e Adaptação de Próteses Auditivas. In: Almeida K, lório MCM. Próteses Auditivas: fundamentos teóricos e aplicações clínicas. 2a ed. São Paulo: Lovise; 2003. p. 305-23.

24. Prates LPCS, lório MCM. Aclimatização: estudo do reconhecimento de fala em usuários de próteses auditivas. Pró-Fono. 2006; 18: 259-66.

25. Dell'Aringa AHB, Esteves MCBN, Dell'Aringa AR, Arruda GV. Resultados da Adaptação do Aparelho de Amplificação Sonora Individual em um Paciente Portador de Neuropatia Auditiva. Arq Int Otorrinolaringol. 2009; 13: 107-10. 